\title{
Approach the Good, Withdraw from the Bad-A Review on Frontal Alpha Asymmetry Measures in Applied Psychological Research
}

\author{
Benny B. Briesemeister, Sascha Tamm, Angela Heine, Arthur M. Jacobs \\ Department of Psychology, Center for Applied Neuroscience, Free University, Berlin, Germany \\ Email: benny.briesemeister@fu-berlin.de \\ Received December $20^{\text {th }}, 2012$; revised January $9^{\text {th }}, 2013$; accepted February $2^{\text {nd }}, 2013$
}

\begin{abstract}
Basic research has established a strong relationship between stimulus induced human motivation for approach-related behavior and left-frontal electrophysiological activity in the alpha band, i.e. frontal alpha asymmetry (FAA). Since approach motivation is also of interest for various fields of applied research, several recent studies investigated the usefulness of FAA as a diagnostic tool of stimulus induced motivational changes. The present review introduces the theory and the methods commonly used in approach/ withdrawal motivation research, and summarizes work on applied FAA with a focus on product design, marketing, brain-computer communication and mental health studies, where approach motivation is of interest. Studies investigating and developing the application of FAA training in the treatment of affective disorders such as major depressive disorder and anxiety disorder are also introduced, highlighting some of the future possibilities.
\end{abstract}

Keywords: Component; Frontal Alpha Asymmetry; Applied Science; Marketing; Depression; EEG

\section{Introduction}

Some 35 years ago, in 1978, a group of researchers around Richard J. Davidson presented a novel finding at the 18th annual meeting of The Society for Psychophysiological Research: Participants who watched portions of a television show were asked to indicate online how much they liked the program, while electroencephalographic (EEG) data was recorded from bilateral frontal and parietal regions. Positively rated TV scenes were associated with greater relative left-hemispheric alpha activation in frontal electrodes, while negatively rated scenes were associated with greater relative right-hemispheric frontal activation. Parietal electrodes, in contrast, did not discriminate between the conditions (Davidson, Schwartz, Saron, Bennett, \& Goleman, 1979). Thirty-five years later, hemispheric asymmetries in the alpha frequency range $(8-12 \mathrm{~Hz})$ over prefrontal electrodes have become widely accepted as a correlate of approach and withdrawal related motivation in basic research (Harmon-Jones, Gable, \& Peterson, 2010; Price, Peterson, \& Harmon-Jones, 2012; Rutherford \& Lindell, 2011), with more and more studies extending the frontal alpha asymmetry (FAA) methodology to diverse fields in applied psychology. The purpose of this non-exhaustive review is to introduce and summarize some of this latter work, beginning with a short introducetion to FAA measurement.

\section{Frontal Alpha Asymmetry, Physiology and the Approach-Withdrawal Hypothesis}

When speaking of FAA, at least two separate perspectives can be identified. On the one hand, FAA refers to a specific way to analyze EEG data, which is described in detail in Allen, Coan, and Nazarian (2004). This perspective will be referred to as the FAA analysis, which involves four processing steps. First, the raw data collected from frontal and prefrontal electrode sites (usually including F3/F4, F7/F8, FP1/FP2) is decomposed into the underlying frequency bands using Fourier transform. Second, assuming that decreased alpha indicates an increase in allocation of cortical resources (Gevins, Smith, McEvoy, \& Yu, 1997) or cortical activation (Allen et al., 2004; Davidson, 1988), frequency bands other than alpha are typically discarded. In a third step, the data is natural-log transformed to reduce positive skewness and kurtosis. Finally, the FAA metric is calculated from these values as difference between right-hemispheric electrodes data minus their left-hemispheric electrodes counterparts $(\ln (\mathrm{R})-\ln (\mathrm{L})$, Allen et al., 2004; Davidson et al., 1979). Thus, positive FFA values indicate larger relative right-hemispheric power, which corresponds to larger cortical resource allocation in the left hemisphere.

Apart from the FAA analysis, there is also the issue of the functional interpretation of FAA on the other hand, which will be referred to as FAA theory. Initial research using the FAA analysis focused on affective manipulations within the stimulus material, reporting positive FAA metrics (interpreted as larger relative left-hemispheric activation) for positive stimuli and negative FAA metrics for negative stimuli (Davidson et al., 1979). This was interpreted as indicators of a systematic relationship between FAA and experienced positive versus negative affect (Harmon-Jones et al., 2010). However, recent research including manipulations that allow for a differentiation of valence and approach-withdrawal motivation (e.g. anger) suggests that only the latter is lateralized in frontal and prefrontal brain regions (Berkman \& Lieberman, 2010; Carver \& Harmon-Jones, 2009). Additional studies on patients with unilateral brain lesions and experiments using transcranial mag- 
netic stimulation (TMS) further support the approach/withdrawal motivation hypothesis (Rutherford \& Lindell, 2011).

Evidence that FAA analysis is linked to larger relative lefthemispheric activation during approach-related motivation but larger right-hemispheric activation during withdrawal-related motivation is convincing. However, the use of FAA analysis as a diagnostic tool, which would be desirable for applied contexts such as marketing research, product design, or brain-computerinterfacing $(\mathrm{BCI})$ is at least problematic. As pointed out by Poldrack (2006), this type of reverse inference, that is to infer motivational states from a given FAA lateralization, would require FAA to be specific for motivational processes only, an issue which, however, is disputable. Several published studies show that the FAA metric is also influenced by unilateral hand contractions (Harmon-Jones et al., 2010), the seating position (Harmon-Jones, Gable \& Price, 2011) or task difficulty in different working memory tasks, even in the absence of emotional distractors (Baldwin \& Penaranda, 2012; Wacker, Chavanon, Leue, \& Stemmler, 2010). None of these variables are explicitly emotionally arousing, even though moderating effects such as greater effort caused by increased task difficulty cannot be excluded (Harmon-Jones et al., 2010). To estimate the specificity of FAA analysis for approach-withdrawal motivation, we follow Ariely and Berns (2010; see also Poldrack, 2006) by determining the posterior probability of the reverse inference using Bayes' theorem. This approach applied to the FAA reverse inference problem is summarized in Table 1. According to the results based on studies published between 2002 and 2012 , the probability of approach/withdrawal motivation given that an FAA effect is observed is 0.79 and the corresponding Bayes factor of 3.75 indicates moderate evidence.

\section{A Few Critical Words Concerning This Review}

Before the published studies that use FAA for diagnostic purposes are summarized in the second part of this review, some methodological and terminological issues need to be addressed. The FAA metric as reviewed here is always used as a relative metric, indicating differences between two groups (e.g. depressed versus non-depressed), two stimuli (e.g. different TV advertisements) or two points in time (e.g. before versus after treatment). Since the FAA metric is a quotient, significant changes (e.g. increased FAA score) can originate from a relative increase in right-hemispheric alpha power, a decrease in left-hemispheric alpha power, or both. Unfortunately, most studies do not provide enough details to distinguish between these three possibilities, which is why effects are reported solely in relation to the FAA theory framework for this review. Moreover, the terms activity and activation are used interchangeably.

\section{Frontal Alpha Asymmetry as a Diagnostic Tool}

Knowing whether a given stimulus elicits approach-related motivation is valuable information for everyone who is designing products or marketing measures to promote products, especially when this information can be collected ahead of a market launch. Therefore, one application for FAA is the improvement of product designs and marketing (section "Frontal alpha asymmetry in design and marketing"). A second major field deals with the application of EEG and FAA analysis in the context of human-computer interactions (section "Frontal alpha asymmetry in Brain-Computer Interfaces"). BCIs are a promising way to help individuals with communication and motor control problems caused by disability (Adams, Bahr \& Moreno, 2008), gaining additional popularity when used as controllers for computer games (Singer, 2008). However, in contrast to design and marketing applications, BCIs are methodologically challenging, since they require FAA feedback in real-time. Finally, a growing literature relating FAA at rest to affective disorders suggests that FAA analysis might reliably distinguish between healthy and clinical populations (section "Frontal alpha asymmetry in the diagnosis of affective disorders"). The following sections are intended to give a comprehensive overview over published work in each of these areas of FAA application.

\section{Frontal Alpha Asymmetry in Design and Marketing}

Studies using FAA to compare different product designs often interpret greater left-frontal activity as an index of pleas-

Table 1.

Inferring motivational states from frontal alpha asymmetry (FAA).

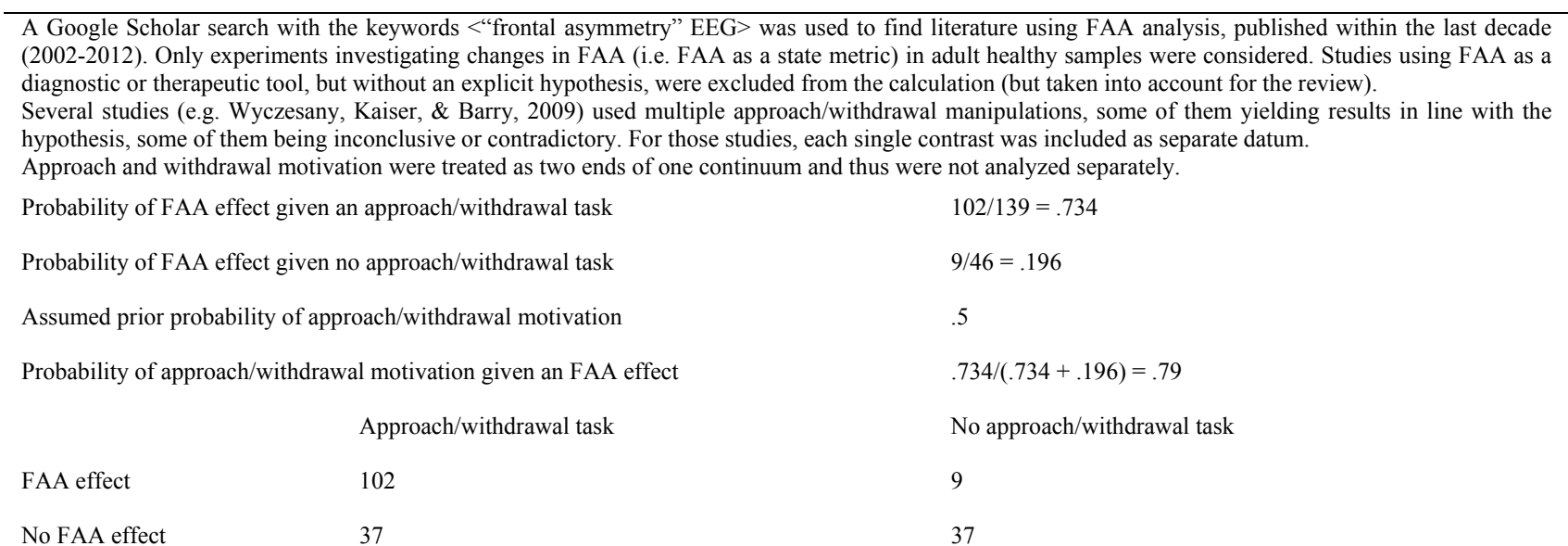

Based on these calculations, the probability of approach/withdrawal motivation given an FAA effect is .79, with a Bayes factor of 3.75 indicating moderate evidence. 
antness or liking. Park and Watanuki (2005), for example, were interested in whether the type of sanitary napkins (mesh versus nonwoven) influences menstrual unpleasantness, as indicated by self-report and greater right hemispheric activity. Results were not consistent, however. Pleasantness ratings indicated that mesh sanitary napkins were less pleasant to wear during follicular phase than nonwoven sanitary napkins, with no differences in menstrual phase or during simulated menstrual bleeding. In contrast, left-hemispheric alpha power was increased after (as compared to before) simulated menstrual bleeding only when wearing nonwoven sanitary napkins, leaving the authors to conclude that the FAA theory might be unsuitable for their purposes. A second study tested the effect of six different ballpens on frontal alpha power, focusing on single-subject analysis (Tomico et al., 2008). Participants were asked to first just look at the pen, to manipulate it in a second step, to use it, and, finally, to manipulate it a second time. This way, a subject-specific alpha power ranking of the six pens in each of the four conditions was obtained. These rankings were combined with self-report data to conclude that the first look at a pen and its manipulation but not its usage were related to the overall product perception.

Another line of design related FAA research focuses on the affective properties of different olfactory aromas. Kline, Blackhart, Woodward, Williams and Schwartz (2000) presented elderly women with seven odors, choosing the most pleasantly (vanilla) and unpleasantly (valerien) rated odor for FAA analysis. As expected, vanilla aroma induced significantly greater relative left-hemispheric activity when compared with valerien or plain water (neutral condition). Hemispheric asymmetries for valerien were not found, however (Kline et al., 2000). Two additional studies focused on the effect of lavender on mood and FAA (Field et al., 2005; Sanders et al., 2002). Sanders et al. (2002) contrasted lavender with rosemary odors, finding the expected increase in relative left-frontal activity for lavender versus baseline, but not for rosemary. This result was replicated by Field et al. (2005), using a lavender shower gel. All of these studies used FAA to investigate and possibly modify the attractiveness of products. Other research, in contrast, focuses on increased marketing efficiency. Neuromarketing (Ariely \& Berns, 2010), that is the application of neuroscientific methods to marketing purposes, has become popular within the last decade, with FAA analysis being one of the most frequently used methods. Vecchiato et al. (2011), for example, presented participants with a 30 min documentary, interrupted by three commercial breaks. Participants were asked which of the TV commercials they remembered and for those remembered, how pleasant they were. Alpha power was then correlated with the pleasantness ratings. As a direct replication of Davidson et al. (1979), pleasant commercials were accompanied by greater right-hemispheric power, while greater left-hemispheric power was observed for unpleasant commercials. Moreover, the FAA score at medial frontal electrodes was significantly correlated with perceived pleasantness. These results were replicated in a very similar setting by Vecchiato et al. (2010), adding skin conductance responses (SCRs) and heart rate variability (HR) as dependent variables.

The Vecchiato studies used a quite complex yet realistic experimental design, embedding the TV commercials into normal program context. Other studies relied on the presentation of TV commercials without additional context. Comparing three different versions of a TV commercial for SONY Bravia ("Balls" aired in 2005, "Paints" aired in 2006 and "Play-Doh" aired in 2007), Ohme, Reykowska, Wiener and Choromanska (2010) used the FAA metric as an indicator of approach tendencies. Each commercial was made of four parts, i.e. the initial emotional part meant to built emotional engagement, a product information blended over the last seconds of the emotional part, presentation of the product itself and finally the presentation of the brand. Using FAA analysis, an overall advantage (i.e. greater FAA score) was found for the balls commercial, driven by the emotional part, the product information and the part where the brand was revealed. The advantage of the brand part was likely to be a carry-over effect, however, caused by the animation of balls surrounding the Sony bravia television in the product presentation scene, which was apparent only in the balls commercial. Consequently, removing the ball animation made the FAA effect disappear (Ohme et al., 2010). In a follow-up study, Ohme, Matukin and Szezurko (2010) further analyzed the balls commercial on a second-by-second basis, in order to identify the scenes which are crucial for emotional involvement. They focused especially on a scene depicting a frog leaping from a drainpipe. Assuming that this scene may have acted as an emotional prime for the rest of the spot based on their initial study, the authors compared two versions of the balls commercial, with and without the frog scene. As predicted, the product information scene following the emotional scenes including the frog caused significantly greater relative lefthemispheric activation than baseline recordings, while no differences from baseline were observed without the frog scene.

A very similar procedure was used in Ohme, Reykowska, Wiener and Choromanska (2009), comparing two versions of a TV commercial for skin care product advertisements. The two spots were almost identical, differing only with respect to a single scene. Behavioral pretests revealed that the difference between the spots was not consciously perceived and did not affect ad recall. When participants of three experimental groups presented with either spot version A, spot version B or neither spot version were given the chance to select the advertised product as a complimentary gift in a shelf test, however, only one spot affected the choice behavior in comparison to the control group. FAA analysis revealed that this spot contained the scene which was associated with larger relative left-hemispheric activation (Ohme et al., 2009).

In contrast to FAA research on advertisements, Burshteyn and Buff (2008) were interested in the perceived differences between private-label brands and manufacturer brands. They assumed that small private-label brands are intentionally designed to resemble manufacturer brands in their product packaging, which should grant them similar attention from the customers. As expected by Burshteyn and Buff, participants indicated no difference between manufacturer-brand and privatelabel brand packaging based on similarity ratings and FAA metrics. Manufacturer-brands were, however, rated as being more familiar.

In summary, previous FAA studies focusing on commercials successfully investigated the emotional engagement of TV viewers. In addition to that, first studies indicate that FAA can provide information concerning the affective value of every day products (Park \& Watanuki, 2005; Tomico et al., 2008) and product packaging (Burshteyn \& Buff, 2008), and suggest that marketing and design can possibly benefit from applied FAA research. 


\section{Frontal Alpha Asymmetry in Brain-Computer Interfaces}

The basic idea behind BCI is to allow for the interaction between computers and humans, who have problems communicating with electronic devices due to disabilities or extreme circumstances (Adams et al., 2008; Molina, Tsoneva, \& Nijholt, 2009). Thus, most of the BCI literature focuses on motor activity and cognitive control, with affective processes being only of secondary interest. At least two BCI applications are assumed to benefit from affective information, however. First, there is evidence that cognitive processes are influenced by emotional processes and mood (Vartak, 2010). In this context, information about a person's affective state can help to make BCI aiming at cognitive processes more efficient by explaining variance that else remains unspecified. Second, BCIs focusing on emotions have caused great interest in the entertainment industry (Gilleade \& Allanson, 2005). Several computer games controlled by the gamers' affective states were developed in recent years, triggered by the availability of affordable BCI based PC controllers (Singer, 2008). Thus, from a theoretical perspective, FAA analysis is a promising BCI methodology.

FAA based BCIs are confronted with two critical methodological problems, however. First, in order to communicate with electronic devices, FAA analysis must be done in real-timewhich is a matter of computational power. Second, unlike most research on FAA, BCI need to operate on the basis of singlesubjects, which increases error variance relative to standard group analyses. This problem is addressed by Winkler, Jäger, Mihajlović and Tsoneva (2010), who analyzed and classified individual data from nine participants on a single trial basis. Only the data of two subjects lead to a classification error below $40 \%$, i.e. a level that is better than chance. These results are somewhat disappointing, but it is important to keep in mind that to evoke emotional responses, Winkler et al. (2010) relied on affective pictures, which have been shown to induce approach or withdrawal related moods only under specific circumstances (Gable \& Harmon-Jones, 2008). In contrast, Huang et al. (2012), using affective video clips, report considerably better classification results. Vartak (2010), finally, demonstrated that the combination of FAA metrics and physiological variables like SCR and HR can identify not only approach/withdrawal motivational tendencies, but discriminate between different discrete emotions on population level. He concludes his paper by stating that the next step should be to get closer to real life applications: "Data should be collected in such environments built for the purpose of training and education" (Vartak, 2010: p. 141). According to Gilleade and Allanson (2005), this already is the case. Affective BCIs receive increased attention from the entertainment industry, and although FAA still plays a minor role with cheaper methods like SCR and HR being preferred, first EEG based videogame controllers are in development (Singer, 2008), which in turn allow the use of FAA metrics.

Taken together, although affective information is of seconddary interest in the development of BCIs, a number of studies indicate that it is possible to reliably classify emotional states based on FAA (Huang et al., 2012; Vartak, 2010), even when being used in single subjects (Huang et al., 2012). With the entertainment industry being interested in affective gaming (Gilleade \& Allanson, 2005), future research in this direction might be profitable. Whether FAA can compete with other currently tested emotion classification approached remains to be investigated (Ko, Yang, \& Sim, 2009; Mühl et al., 2011).

\section{Frontal Alpha Asymmetry in the Diagnosis of Affective Disorders}

While the studies reviewed so far focused on FAA as a measure of induced approach/withdrawal motivation changes, the most prominent line of applied FAA research investigates the relationship between FAA at rest and affective disorders, especially focusing on the major depressive disorder (MDD). Roughly following Thibodeau, Jorgensen and Kim's (2006) excellent meta-analysis on the matter, this part of this review will focus on four aspects: First, studies supporting the claim that FAA can differentiate between participants currently suffering from MDD and healthy controls are introduced. Second, evidence for FAA as an indicator of risk for MDD is reviewed. Third, studies testing FAA as an indicator for therapeutic success are discussed. This aspect was not considered in Thibodeau et al. (2006) and is thus discussed more extensively. Finally, the problem of comorbidity of MDD with other affective disorders such as the anxiety disorder is addressed.

MDD is one of the most prevalent affective disorders at present (Kurth, 2012), which entails the importance of effective diagnostic tools. FAA analysis seems to reliably discriminate between depressed and control subjects, by revealing decreased left-frontal lateralization for depressed subjects in the presence (Stewart, Coan, Towers, \& Allen, 2011) and absence of external stimulation (Allen, Urry, Hitt, \& Coan, 2004; Gotlieb, Ranganath, \& Rosenfeld, 1998). Allen et al. (2011) document internal consistency and stability of FAA scores in depressed participants over a time period of altogether 16 weeks, and Rosenfeld, Baehr, Baehr, Gotlib and Ranganath (1996) report therapy-induced affective changes in five depressed participants being significantly related to the FAA score. Based on altogether 31 studies using 59 separate tests, Thibodeau et al. (2006) conclude that the existing data suggest a relation between depression and reduced relative left-frontal activation, with age, recording duration and operationalization of depresssion as important moderating factors.

However, FAA is not only sensitive to the current status of patients (depressed versus non-depressed), but also differentiates high from low depression risk groups. Tomarken, Dichter, Garber and Simien (2004), who contrasted adolescents whose mothers had a history of depression (high risk group) with adolescents with never-depressed mothers (low risk group), found decreased relative left-frontal activation for the high risk group. Bruder, Tenke, Warner and Weissman (2005) basically replicated this result for grandchildren of depressed (high risk) versus never-depressed (low risk) parents and grandparents ${ }^{1}$.

Complementing this line of research, FAA analysis may also be used as an indicator of the effects of therapeutic intervention. Based on FAA theory, a decrease in depressive symptoms and an increase in well-being should be accompanied by an increase in left-frontal activity, given that reduced left-frontal activity is characteristic for MDD - which is supported by a study by Segrave et al. (2011). Contrasting depressed participants with and without current medication, significantly larger relative left-frontal activity was found for participants taking antidepressants, even though both groups did not differ with respect

\footnotetext{
${ }^{1}$ The effect was significant over parietal rather than frontal electrodes, however.
} 
to the severity of symptoms. It should be noted, however, that these results stem from a between-subject design, which means that these differences may also be explained by confounding between-subject variables. Rosenfeld et al. (1996), in contrast, report strong correlations between the FAA metric and affect change in depressed participants. A comparison of depressed participants before and after a 12 week antidepressant treatment replicated this result (Bruder et al., 2001), identifying lateralized alpha power as significant predictor of treatment outcome in a multiple regression analysis. Moreover, participants who did not respond to medication showed greater right-hemispheric activation in FAA analysis than participants who did, suggesting that FAA might be able to predict medication success.

While most FAA studies on depression report promising results, with the meta-analysis by Thibodeau et al. (2006) revealing moderately large effect sizes, several studies also report null effects (Thibodeau et al., 2006). Since reliable results are of great importance for diagnostic purposes, first studies try to improve the diagnostic value of FAA by developing advanced FAA analysis techniques. Segrave et al. (2011), for example, contrasted FAA data within a predefined alpha range $(8-13 \mathrm{~Hz})$ with FAA data using individualized alpha bandwidths. This approach aims at reducing between-subject variance not related to depression. No significant differences between the two approaches were found, but since depressed and normal control subjects did not differ in any FAA analysis, these results are difficult to interpret anyway. Baehr, Rosenfeld, Baehr and Earnest (1998) took a different approach, comparing the predictive power of the standard FAA score with the percentage of time where the asymmetry score was greater than 0 , which indicates relative left-hemispheric activity. Both measures discriminated between depressed participants and controls, but the percentage score was the better predictor on a single-subject level with a discrimination rate of $83 \%$ (versus $54 \%$ for FAA).

According to FAA theory, hemispheric asymmetries are an indicator of approach/withdrawal motivation and thus not specific for a single affective disorder. This is probably the greatest challenge for FAA as a diagnostic tool. MDD is typically characterized by reduced positive affect and a loss of interest and energy, which - in terms of the approach/withdrawal framework-means reduced approach motivation causing reduced left-frontal activity. Other affective disorders, however, should affect FAA in similar ways. Anxiety disorder, as a prominent example, is characterized by strongly increased withdrawal motivation, and should, thus, affect FAA analysis. In fact, Thibodeau et al. (2006) summarize several studies which indicate relative right-hemispheric activation for anxiety patients. Moscovitch et al. (2011) report that successful cognitive behavioral therapy for socially anxious patients is accompanied by a shift from relative right-hemispheric to relative left-hemispheric activity, with relative left FAA at rest before the treatment predicting greater reduction and an overall lower level of social anxiety after successful treatment. A comparison of depressed patients with patients diagnosed with post-traumatic stress disorder-both disorders being associated with reduced approach/ increased withdrawal motivation - and healthy controls found differential effects for both disorders (Kemp et al., 2010). Keune et al. (2011), in contrast, focused on subjects with increased approach motivation, namely patients with attention deficit hyperactivity disorder (ADHD). In comparison to healthy controls, ADHD patients were characterized by increased leftfrontal activity. Moreover, ADHD patients scored significantly higher on the hostility subscale of an aggression questionnaire, which further supports the approach/withdrawal FAA theory.

\section{Frontal Alpha Asymmetry as a Therapeutic Tool}

Thibodeau et al. (2006: p. 728) conclude their meta-analysis by saying "that both depression and anxiety are meaningfully related to relative right-sided resting frontal EEG asymmetry." Spronk, Arns, Bootsma, van Ruth and Fitzgerald (2008) even suggest a causal relationship, showing remission of depressive symptoms after repeated use of repetitive TMS stimulation over left-frontal sides. Rosenfeld, Cha, Blair and Gotlib (1995) document that FAA can be modulated by simple training, and Allen, Harmon-Jones and Cavender (2001) report that the manipulation of FAA affects emotional responses in healthy participants. Taken together, these studies suggest that FAA might be able to not only diagnose but actually affect depressive symptoms via neurofeedback. Initial studies training depressed participants to increase their relative left-hemispheric activity document that depressive symptoms indeed decrease after neurofeedback training (Baehr, Rosenfeld, \& Baehr, 1997; Hammond, 2000, Rosenfeld et al., 1996), even when participants were under age (Earnest, 1999).

Baehr et al. (1997) additionally report two case studies where both patients received medication and psychotherapy before the neurofeedback study had started, but neither of them made significant progress until neurofeedback was introduced. FAA treatment not only affected the depressive symptoms, but also improved flexibility in thinking and induced a positive outlook for the future compared to before treatment (Baehr et al., 1997). Choi et al. (2010) basically replicated these results, contrasting neurofeedback with psychotherapy placebo in depressed patients. Neurofeedback exceeded psychotherapy placebo in terms of decreased depressive symptoms, enhanced executive functions and enhanced verbal fluency. Moreover, benefits from neurofeedback seem to be stable long after the actual training has ended, as indicated by a 5 year follow-up study (Baehr, Rosenfeld, \& Baehr, 2001).

Given the bilateral relationship between FAA and depression-depression is characterized by decreased left-frontal activity, and manipulations within FAA affect depressive symptoms - Choi et al. (2010: p. 49) conclude that the result of their study "suggested a causal relationship between the asymmetric frontal activity and depressive symptoms". An earlier paper by Davidson (1998), in contrast, favors a less deterministic view of FAA, suggesting that "differences in prefrontal activation are neither necessary nor sufficient for the production of a specific type of affective style or psychopathology" but should be seen as "diatheses that bias a person's affective style, and then in turn modulate an individual's vulnerability to develop depression" (Davidson, 1998: p. 608).

Irrespective of the theoretical background, two recent studies document that FAA training is not only effective in depression therapy, but generally helps patients suffering from disorders that affect approach/withdrawal motivation (Hammond, 2005; Kerson, Sherman, \& Kozlowski, 2009). The presented literature thus strongly suggests that FAA can be a successful therapeutic tool.

\section{Conclusion}

Approach/withdrawal motivation as a theoretical construct can be useful in several applied contexts. FAA analysis has 
been established as a reliable index influenced by approach/ withdrawal motivation, and the analysis of the probability of approach/withdrawal motivation given an FAA effect (see Table 1) revealed a substantial relationship (Kass \& Raftery, 1995), thus allowing for a cautious interpretation of FAA effects in the absence of an a-priori hypothesis.

Keeping in mind that the application of FAA in applied research is still in its infancy, the present review was meant to introduce and summarize some of the first studies documenting the various possibilities of FAA application. Based on the reviewed studies, FAA analysis seems to be a promising diagnosetic tool at least in marketing and in the detection of affective disorders. Moreover, assuming that affective gaming continues to gain popularity, $\mathrm{BCI}$ might also profit from FAA analysis.

Finally, based on the existing literature, FAA seems to significantly contribute to the diagnosis and-even more important- treatment of affective disorders, such as depression and anxiety. Even though this area of application still needs further systematic research including experimental studies on larger samples, the existing results are promising. Given that the casestudy by Baehr et al. (1997) indicates that FAA neurofeedback successfully complements medication and psychotherapy, and given that Choi et al. (2010) report a neurofeedback-related increase in several cognitive functions exceeding the expected decrease in depressive symptoms, the diagnosis and treatment of affective disorders is a field which will greatly benefit from FAA application in the future.

\section{Acknowledgements}

This work was financially supported by the German Federal Ministry of Education and Research (BMBF) under the research initiative 'ForMaT' (03FO2912).

\section{REFERENCES}

Adams, R. G., Bahr, G. S., \& Moreno, B. (2008). Brain computer interfaces: Psychology and pragmatic perspectives for the future. Proceedings of the AISB 2008 Symposium on Brain Computer Interfaces and Human Computer Interaction: A Convergence of Ideas, 5, 1-6.

Allen, J. J. B., Coan, J. A., \& Nazarian, M. (2004). Issues and assumptions on the road from raw signals to metrics of frontal EEG asymmetry in emotion. Biological Psychology, 67, 183-218. doi:10.1016/j.biopsycho.2004.03.007

Allen, J. J. B., Harmon-Jones, E., \& Cavender, J. H. (2001) Manipulation of frontal EEG asymmetry through biofeedback alters self-reported emotional responses and facial EMG. Psychophysiology, 38, 685-693. doi:10.1111/1469-8986.3840685

Allen, J. J. B., Urry, H. L., Hitt, S. K., \& Coan, J. A. (2004). The stability of resting frontal electroencephalographic asymmetry in depresssion. Psychophysiology, 41, 269-280. doi:10.1111/j.1469-8986.2003.00149.x

Ariely, D., \& Berns, G. S. (2010). Neuromarketing: The hope and hype of neuroimaging in business. Nature Reviews Neuroscience, 11, 284292. doi: $10.1038 / \mathrm{nrn} 2795$

Baehr, E., Rosenfeld, J. P., \& Baehr, R. (1997). The clinical use of an alpha asymmetry protocol in the neurofeedback treatment of depresssion. Journal of Neurotherapy: Investigations in Neuromodulation, Neurofeedback and Applied Neuroscience, 2, 10-23.

Baehr, E., Rosenfeld, J. P., \& Baehr, R. (2001). Clinical use of an alpha asymmetry neurofeedback protocol in the treatment of mood disorders: Follow-up study one to five years post therapy. Journal of Neurtherapy, 4, 11-18. doi:10.1300/J184v04n04 03

Baehr, E., Rosenfeld, J. P., Baehr, R., \& Earnest, C. (1998). Comparison of two EEG asymmetry indices in depressed patients vs. normal controls. International Journal of Psychophysiology, 31, 89-92. doi:10.1016/S0167-8760(98)00041-5

Baldwin, C. L., \& Penaranda, B. N. (2012). Adaptive training using an artificial neural network and EEG metrics for within- and cross-task workload classification. NeuroImage, 59, 48-56. doi:10.1016/j.neuroimage.2011.07.047

Berkman, E. T., \& Lieberman, M. D. (2010). Appoaching the bad and avoiding the good: Lateral prefrontal cortical asymmetry distinguishes between action and valence. Journal of Cognitive Neuroscience, 22, 1970-1979. doi:10.1162/jocn.2009.21317

Bruder, G. E., Stewart, J. W., Tenke, C. E., McGrath, P. J., Leite, P., Bhattacharya, N., \& Quitkin, F. M. (2001). Electroencephalographic and perceptual asymmetry differences between responders and nonresponders to an SSRI antidepressant. Biological Psychiatry, 49, 416-425. doi:10.1016/S0006-3223(00)01016-7

Bruder, G. E., Tenke, C. E., Warner, V., \& Weissman, M. M. (2005). Grandchildren at high and low risk for depression differ in EEG measures of regional brain asymmetry. Biological Psychiatry, 62, 1317-1323. doi:10.1016/j.biopsych.2006.12.006

Burshteyn, D., \& Buff, C. L. (2008). Provate-label brands, manufacturer brands, and the quest for stimulus generalization: An EEG analysis of frontal cortex response. Review of Business Research, 8, 9296.

Carver, C. S., \& Harmon-Jones, E. (2009). Anger is an approach-related affect: Evidence and implications. Psychological Bulletin, 135, 183-204. doi:10.1037/a0013965

Choi, S. W., Chi, E. E., Chung, S. Y., Kim, J. W., Ahn, C. Y., \& Kim, H. T. (2010). Is alpha wave neurofeedback effective with randomized clinical trials in depression? A pilot study. Neuropsychobiology, 63, 43-51. doi:10.1159/000322290

Davidson, R. J. (1988). EEG measures of cerebral asymmetry: Conceptual and methodological issues. International Journal of Neuroscience, 39, 71-89. doi:10.3109/00207458808985694

Davidson, R. J. (1998). Anterior electrophysiological asymmetries, emotion, and depression: Conceptual and methodological conundrums. Psychophysiology, 35, 607-614. doi: $10.1017 /$ S0048577298000134

Davidson, R. J., Schwartz, G. E., Saron, C., Bennett, J., \& Goleman, D. J. (1979). Frontal versus parietal EEG asymmetry during positive and negative affect. Psychophysiology, 16, 202-203.

Earnest, C. (1999). Singe case study of EEG asymmetry biofeedback for depression: An independent replication in an adolescent. Journal of Neurotherapy: Investigations in Neuromodulation, Neurofeedback and Applied Neuroscience, 3, 28-35.

Field, T., Diego, M., Hernandez-Reif, M., Cisneros, W., Feijo, L., Vera, Y., \& He, Q. C. (2005). Lavender fragrance cleansing gel effects on relaxation. International Journal of Neuroscience, 115, 207-222. doi: $10.1080 / 00207450590519175$

Gable, P., \& Harmon-Jones, E. (2008). Relative left frontal activation to appetitive stimuli: Considering the role of individual differences. Psychophysiology, 45, 275-278. doi:10.1111/j.1469-8986.2007.00627.x

Gevins, A., Smith, M. E., McEvoy, L., \& Yu, D. (1997). High-resolution EEG mapping of cortical activation related to working memory: Effects of task difficulty, type of processing, and practice. Cerebral Cortex, 7, 374-385. doi:10.1093/cercor/7.4.374

Gilleade, K. M., \& Allanson, J. (2005). Affective videogames and modes of affective gaming: Assist me, challenge me, emote me. Proceedings of DiGRA 2005 Conference: Changing Views. Worlds in Play, 1-7.

Gotlieb, I. H., Ranganath, C., \& Rosenfeld, P. (1998). Frontal EEG alpha asymmetry, depression, and cognitive functioning. Cognition \& Emotion, 12, 449-478. doi:10.1080/026999398379673

Hammond, D. C. (2000). Neurofeedback treatment of depression with the Roshi. Journal of Neurotherapy: Investigations in Neuromodulation, Neurofeedback and Applied Neuroscience, 4, 45-56.

Hammond, D. C. (2005). Neurofeedback treatment of depression and anxiety. Journal of Adult Development, 12, 131-137. doi:10.1007/s10804-005-7029-5

Harmon-Jones, E., Gable, P. A., \& Peterson, C. K. (2010). The role of asymmetric cortical activity in emotion-related phenomena: A review 
and update. Biological Psychology, 84, 451-462.

doi:10.1016/j.biopsycho.2009.08.010

Harmon-Jones, E., Gable, P. A., \& Price, T. F. (2011). Leaning embodies desire: Evidence that leaning forward increases relative left frontal activation to appetitive stimuli. Biological Psychology, 87, 311313. doi:10.1016/j.biopsycho.2011.03.009

Huang, D., Zhang, H., Ang, K., Guan, C., Pan, Y., Wang, C., \& Yu, J. (2012). Fast emotion detection from EEG using asymmetric spatial filtering. Proceedings of the International Conference on Acoustics, Speech and Signal Processing (ICASSP), 589-592.

Kass, R. E., \& Raftery, A. E. (1995). Bayes factors. Journal of the American Statistical Association, 90, 773-795. doi:10.1080/01621459.1995.10476572

Kemp, A. H., Griffiths, K., Felmingham, K. L., Shankman, S. A., Drinkenburg, W., Arns, M., \& Bryant, R. A. (2010). Disorder specificity despite comorbidity: Resting EEG alpha asymmetry in major depressive disorder and post-traumatic stress disorder. Biological Psychology, 85, 350-354. doi:10.1016/j.biopsycho.2010.08.001

Kerson, C., Sherman, R. A., \& Kozlowski, G. P. (2009). Alpha suppression and symmetry training for generalized anxiety symptoms. Journal of Neurotherapy, 13, 146-155.

doi:10.1080/10874200903107405

Keune, P. M., Schönenberg, M., Wyckoff, S., Mayer, K., Riemann, S., Hautzinger, M., \& Strehl, U. (2011). Frontal alpha-asymmetry in adults with attention deficit hyperactivity disorder: Replication and specification. Biological Psychology, 87, 306-310. doi:10.1016/j.biopsycho.2011.02.023

Kline, J. P., Blackhart, G. C., Woodward, K. M., Williams, S. R., \& Schwartz, G. E. R. (2000). Anterior electroencephalographic asymmetry changes in elderly women in response to a pleasant and an unpleasant odor. Biological Psychology, 52, 241-250. doi:10.1016/S0301-0511(99)00046-0

Ko, K.-E., Yang, H. C., \& Sim, K.-B. (2009). Emotion recognition using EEG signals with relative power values and Bayesian network. International Journal of Control, Automation, and Systems, 7, 865870. doi:10.1007/s12555-009-0521-0

Kurth, B.-M. (2012). Erste Ergebnisse aus der "Studie zur Gesundheit Erwachsener in Deutschland" (DEGS). Bundesgesundheitsblatt, 55, 980-990. doi:10.1007/s00103-012-1504-5

Molina, G. G., Tsoneva, T., \& Nijholt, A. (2009). Emotional braincomputer interfaces. Proceedings of the International Conference on Affective Computing and Intelligent Interaction, 1-9.

Moscovitch, D. A., Santesso, D. L., Miskovic, V., McCabe, R. E., Antony, M. M., \& Schmidt, L. A. (2011). Frontal EEG asymmetry and symptom response to cognitive behavioral therapy in patients with social anxiety disorder. Biological Psychology, 87, 379-385. doi:10.1016/j.biopsycho.2011.04.009

Mühl, C., Brouwer, A.-M., van Wouwe, N. C., van den Broek, E. L., Nijboer, F., \& Heylen, D. K. J. (2011). Modality-specific affective responses and their implications for affective BCI. Proceedings of the Fifth International Brain-Computer Interface Conference 2011, 120-123.

Ohme, R., Matukin, M., \& Szezurko, T. (2010). Neurophysiology uncovers secrets of TV commercials. Der markt, 49, 133-142. doi:10.1007/s12642-010-0034-7

Ohme, R., Reykowska, D., Wiener, D., \& Choromanska, A. (2009). Analysis of neurophysiological reactions to advertising stimuli by means of EEG and galvanic skin response measures. Journal of Neuroscience, Psychology, and Economics, 2, 21-31. doi: $10.1037 / \mathrm{a} 0015462$

Ohme, R., Reykowska, D., Wiener, D., \& Choromanska, A. (2010). Application of frontal EEG asymmetry to advertising research. Journal of Economic Psychology, 31, 785-793. doi:10.1016/j.joep.2010.03.008

Park, M.-K., \& Watanuki S. (2005). Electroencephalograhic responses and subjective evaluation on unpleasantness induced by sanitary napkins. Journal of Physiological Anthropological and Applied Human Science, 24, 67-71. doi:10.2114/jpa.24.67

Poldrack, R. A. (2006). Can cognitive processes be inferred from neuroimaging data? Trends in Cognitive Sciences, 10, 59-63. doi:10.1016/j.tics.2005.12.004

Price, T. F., Peterson, C. K., \& Harmon-Jones, E. (2012). The emotive neuroscience of embodiment. Motivation and Emotion, 36, 27-37. doi:10.1007/s11031-011-9258-1

Rosenfeld, J. P., Baehr, E., Baehr, R., Gotlib, I. H., \& Ranganath, C. (1996). Preliminary evidence that daily changes in frontal alpha asymmetry correlate with changes in affect in therapy sessions. International Journal of Psychophysiology, 23, 137-141.

doi:10.1016/0167-8760(96)00037-2

Rosenfeld, J. P., Cha, G., Blair, T., \& Gotlib, I. H. (1995). Operant (biofeedback) control of left-right frontal alpha power differences: Potential neurotherapy for affective disorders. Biofeedback and SelfRegulation, 20, 241-258. doi:10.1007/BF01474516

Rutherford, H. J. V., \& Lindell, A. K. (2011). Thriving and surviving: Approach and avoidance motivation and lateralization. Emotion Review, 3, 333-343. doi:10.1177/1754073911402392

Sanders, C., Diego, M., Fernandez, M., Field, T., Hernandez-Reif, M. $\&$ Roca, A. (2002). EEG asymmetry responses to lavender and rosemary aromas in adults and infants. International Journal of Neuroscience, 112, 1305-1320. doi:10.1080/00207450290158214

Segrave, R. A., Cooper, N. R., Thomson, R. H., Croft, R. J., Sheppard, D. M., \& Fitzgerald, P. B. (2011). Individualized alpha activity and frontal asymmetry in major depression. Clinical EEG and Neuroscience, 42, 45-52. doi: $10.1177 / 155005941104200110$

Singer, E. (2008). Brain Games. Technology Review, 111, 82-84.

Spronk, D., Arns, M., Bootsma, A., van Ruth, R., \& Fitzgerald, P. B. (2008). Long term effects of left frontal rTMS on EEG and ERPs in patients with depression. Clinical EEG and Neuroscience, 39, 118124. doi: $10.1177 / 155005940803900305$

Stewart, J. L., Coan, J. A., Towers, D. N., \& Allen, J. J. B. (2011). Frontal EEG asymmetry during emotional challenge differentiates individuals with and without major depressive disorder. Journal of Affective Disorders, 129, 167-174. doi:10.1016/j.jad.2010.08.029

Thibodeau, R., Jorgensen. R. S., \& Kim, S. (2006). Depression, anxiety, and resting frontal EEG asymmetry: A meta-analytic review. Journal of Abnormal Psychology, 115, 715-729. doi:10.1037/0021-843X.115.4.715

Tomarken, A. J., Dichter, G. S., Garber, J., \& Simien, C. (2004). Resting frontal brain activity: Linkages to maternal depression and socioeconomic status among adolescents. Biological Psychology, 67, 77102. doi:10.1016/j.biopsycho.2004.03.011

Tomico, O., Mizutani, N., Levy, P., Takahiro, Y., Cho, Y., \& Yamanaka, T. (2008). Kansei physiological measurements and constructivist psychological explorations for approaching user subjective experience during and after product usage. Proceedings of the DESIGN 2008, 10th International Design Conference. Croatia, 529-536

Vartak, A. A. (2010). Biosignal processing challenges in emotion recognition for adaptive learning. Ph.D. Thesis, Orlando, FL: University of Central 2010.

Vecchiato, G., Toppi, J., Astolfi, L., De Vico Fallani, F., Cincotti, F., Mattia, D., \& Babiloni, F. (2010). Changes in brain activity during the observation of TV commercials by using EEG, GSR and HR measurements. Brain Topography, 23, 165-179. doi:10.1007/s10548-009-0127-0

Vecchiato, G., Toppi, J., Astolfi, L., De Vico Fallani, F., Cincotti, F., Mattia, D., \& Babiloni, F. (2011). Spectral EEG frontal asymmetries correlate with the experienced pleasantness of TV commercial advertisements. Medical \& Biological Engineering \& Computing, 49, 579583. doi:10.1007/s11517-011-0747-X

Wacker, J., Chavanon, M.-L., Leue, A., \& Stemmler, G. (2010). Trait BIS predicts alpha asymmetry and P300 in a Go/No-Go task. European Journal of Personality, 24, 85-105.

Winkler, I., Jäger, M., Mihajlović, V., \& Tsoneva, T. (2010). Frontal EEG asymmetry based classification of emotional valence using common spatial patterns. World Academy of Science, Engineering and Technology, 45, 373-378.

Wyczesany, M., Kaiser, J., \& Barry, R. J. (2009). Cortical lateralization patterns related to self-estimation of emotional state. Acta Neurobiologiae Experimentalis, 69, 526-536. 